\title{
Methylphenidate Enhances Working Memory by Modulating Discrete Frontal and Parietal Lobe Regions in the Human Brain
}

\author{
Mitul A. Mehta, ${ }^{1}$ Adrian M. Owen, ${ }^{3}$ Barbara J. Sahakian, ${ }^{1}$ Nahal Mavaddat, ${ }^{1}$ John D. Pickard, ${ }^{2}$ and \\ Trevor W. Robbins ${ }^{4}$ \\ ${ }^{1}$ University of Cambridge, Department of Psychiatry, and 2Wolfson Brain Imaging Centre, Addenbrooke's Hospital, \\ Cambridge, CB2 2QQ, United Kingdom, ${ }^{3}$ Medical Research Council Cognition and Brain Sciences Unit, Cambridge, CB2 \\ 2EF, United Kingdom, and 4University of Cambridge, Department of Experimental Psychology, Downing Site, Cambridge \\ CB2 3EB, United Kingdom
}

\begin{abstract}
The indirect catecholamine agonist methylphenidate (Ritalin) is the drug treatment of choice in attention deficit/hyperactivity disorder (AD/HD), one of the most common behavioral disorders of childhood (DSM-IV), although symptoms may persist into adulthood. Methylphenidate can enhance cognitive performance in adults and children diagnosed with AD/HD (Kempton et al., 1999; Riordan et al., 1999) and also in normal human volunteers on tasks sensitive to frontal lobe damage, including aspects of spatial working memory (SWM) performance (Elliott et al., 1997). The present study investigated changes in regional cerebral blood flow (rCBF) induced by methylphenidate during performance of a self-ordered SWM task to define the neuro-
\end{abstract}

anatomical loci of the beneficial effect of the drug. The results show that the methylphenidate-induced improvements in working memory performance occur with task-related reductions in rCBF in the dorsolateral prefrontal cortex and posterior parietal cortex. The beneficial effects of methylphenidate on working memory were greatest in the subjects with lower baseline working memory capacity. This is to our knowledge the first demonstration of a localization of a drug-induced improvement in SWM performance in humans and has relevance for understanding the treatment of AD/HD.

Key words: cognition; dopamine; humans; methylphenidate; neuroimaging; Ritalin; stimulant; working memory
Methylphenidate is a stimulant drug related to amphetamine that acts to increase the synaptic concentration of dopamine and noradrenaline (catecholamines) by blocking their reuptake (Scheel-Kruger et al., 1977; Seeman and Madras, 1998). Both of these neurotransmitters are known to affect performance of spatial working memory (SWM) tasks in monkeys (Arnsten, 1997) and humans (Coull et al., 1995; Elliott et al., 1997). Local injection and iontophoretic application of specific dopaminergic (Bernardi et al., 1982; Sawaguchi et al., 1988) and noradrenergic agents (Arnsten and Goldman-Rakic, 1985) as well as electrophysiological measures (Bernardi et al., 1982) in the monkey have identified the lateral prefrontal cortex as a critical region by which these monoamine systems influence SWM processes. In addition, in humans, methylphenidate enhances performance of the SWM task used in the present study (Elliott et al., 1997). However, the neural (cortical or subcortical) localization of the effects of stimulant medications, such as methylphenidate, on working memory performance has not been determined in human subjects. The prefrontal and temporal cortices can be hypothesized to be possible neuromodulatory sites of action because damage to these cortical areas impairs self-ordered SWM performance (Owen et al., 1996a), and activations of the dorsolateral and ventrolateral

Received Oct. 26, 1999; revised Jan. 7, 2000; accepted Jan. 19, 2000.

This work was supported by a Wellcome Trust Programme Grant to T.W.R., B. J. Everitt, A. C. Roberts, and B.J.S. M.A.M. was supported by a Medical Research Council (MRC) Studentship. We thank Dr. David A. Menon for clinical advice, Dr. Wim Reidel for assistance, and Dr. Matthew Brett for discussion. This research was completed within the MRC Cooperative group in Brain, Behavior, and Neuropsychiatry.

Correspondence should be addressed to Dr. Barbara Sahakian at University of Cambridge, Department of Psychiatry, Level E4, Addenbrooke's Hospital, Cambridge, CB2 2QQ, UK. E-mail: jenny.hall@msexc.addenbrookes.anglox.nhs.uk.

Copyright (C) 2000 Society for Neuroscience $\quad 0270-6474 / 00 / 200001-\bullet \$ 15.00 / 0$ prefrontal cortex (in addition to PPC) are observed during functional neuroimaging (Owen et al., 1996b). In humans, direct approaches aimed at defining the neuroanatomical site of performance modulation by methylphenidate in vivo have only become possible with the advent of functional neuroimaging techniques.

Thus, we conducted the present study to examine the effects of methylphenidate on regional cerebral blood flow ( $\mathrm{rCBF}$ ) in normal volunteers using positron emission tomography (PET). Based on the results of previous studies (Mattay et al., 1996; Owen et al., 1996b; Elliott et al., 1997), we predicted that methylphenidate would enhance spatial working memory performance and that this would be accompanied by changes in $\mathrm{rCBF}$ in regions of the frontal and parietal lobes known to be activated by the task (Owen et al., 1996b).

\section{MATERIALS AND METHODS}

Procedure. The study protocol for this investigation was approved by the Cambridge Local Research Ethics Committee and the Administration of Radioactive Substances Advisory Council. Written informed consent was obtained for all subjects. For the study, 10 healthy right-handed male

This article is published in The Journal of Neuroscience, Rapid Communications Section, which publishes brief, peerreviewed papers online, not in print. Rapid Communications are posted online approximately one month earlier than they would appear if printed. They are listed in the Table of Contents of the next open issue of JNeurosci. Cite this article as: JNeurosci, 2000, 20:RC65 (1-6). The publication date is the date of posting online at www.jneurosci.org.

http://www.jneurosci.org/cgi/content/full/4029 
Table 1. Stereotaxic coordinates of significant changes in rCBF in the drug condition compared with the placebo conditions

\begin{tabular}{lcccc} 
& \multicolumn{3}{l}{ Stereotaxic coordinates } & \\
\cline { 2 - 4 } Area of activation (BA) & $x$ & $y$ & $z$ & $Z$ score \\
\hline Increases & 10 & -48 & -28 & 5.80 \\
$\quad$ Right cerebellum & & & & \\
Decreases & -24 & 8 & -20 & 6.64 \\
$\quad$ Left temporal pole (28) & 18 & 8 & -20 & 5.87 \\
Right temporal pole (28) & 22 & 62 & -8 & 3.84 \\
Frontal pole (10) & -34 & -86 & 12 & 4.38 \\
Primary visual cortex (18) & 68 & -36 & -8 & 3.72 \\
$\quad$ Medial temporal gyrus (21) & & & & \\
\end{tabular}

$\overline{\text { Activation foci represent peaks of corrected statistically significant changes in normalized rCBF (see Materials and }}$ Methods). The stereotaxic coordinates are expressed in millimeters. $x$, Medial-to-lateral distance relative to the midline (positive values $=$ right hemisphere); $y$, anterior-to-posterior distance relative to the anterior commissure (positive $=$ anterior); $z$, superior-to-inferior distance relative to the anterior-posterior commissure line (positive $=$ superior).

volunteers (mean age, 34.8 years; SD, 3.40) were seen on two occasions separated by 2 weeks. On each session volunteers were given either $40 \mathrm{mg}$ of methylphenidate or placebo (lactose) presented in identical capsules. Imaging commenced $\sim 90$ min after ingestion of the capsule to maximize the levels of drug during the scans (Gualtieri et al., 1982). Before scans or tablet ingestion on session 1 , subjects were given a brief baseline assessment with the digit span test from the Weschler Adult Intelligence Scale-Revised, the National Adult Reading Test, and the spatial span test from the CANTAB battery (Owen et al., 1996b). In addition, subjects were trained on a simple version (four and six boxes) of the SWM task used during the activation scans. On each test session, cardiovascular measures and a set of 16 visual analog scale ratings (Bond and Lader, 1974) were taken before tablet ingestion, after scan 3 or 4, and after scan 6. For each session, six PET scans (each of $90 \mathrm{sec}$ data acquisition) were obtained for each subject, two for each of three task conditions (see below). The order of administration of the tasks was randomized across the six scans for each subject. The General Electric Advance system was used to measure rCBF. For each scan subjects received a $20 \mathrm{sec}$ intravenous bolus of $\mathrm{H}_{2}{ }^{15} \mathrm{O}$ through a cannula in the left arm at a concentration of $300 \mathrm{Mbq} / \mathrm{ml}$ and flow rate of $10 \mathrm{ml} / \mathrm{min}$ before data acquisition.

Cognitive task. The two task conditions and control condition used were identical to those described by Owen et al. (1996b). For the task conditions, subjects were presented either six ("easy") or twelve ("difficult") red circles on a touch-sensitive computer screen suspended above the scanner. For each problem, subjects were required to search through the array of red circles for blue tokens by touching each one to reveal its contents. The goal was to find all of the blue tokens, which were hidden behind the red circles. The key instruction was that, once a blue token had been found behind a particular red circle, that circle would not be used again to hide a token. Each circle was only used once to hide a token, and therefore two types of error were possible. A between-search error occurred when a subject returned to a circle in which they had previously found a blue token, and a within-search error occurred when a subject returned to a circle within the same search. The performance measure of errors described in Results refers to between-search errors. Subjects performed the task continually for $30 \mathrm{sec}$ before, and for the 90 sec duration of, scan acquisition. Over this period of time, subjects performed between three and five task problems. The control condition was designed to have similar visual, spatial, and motor requirements as both of the task conditions. Eight circles were, therefore, presented on the computer screen, and the central one changed color at a rate of approximately once per second. On the color change, subjects were required to touch the central circle with the same finger used for the memory tasks.

Data analysis. Initially, individual three-dimensional (3-D) magnetic resonance imaging $(\mathrm{MRI})$ was obtained (whole brain, $256 \times 256 \times 128$ pixels) using a $0.5 \mathrm{~T}$ system. MRI images were resliced to be coregistered with the PET data to allow for anatomical localization of regions with statistically significant changes in rCBF between conditions. PET data were realigned (to individual MRI scans and to a standard brain), normalized (for global CBF), smoothed (using an isotropic Gaussian kernel of $16 \mathrm{~mm}$ ), and analyzed using the Statistical Parametric Mapping 96 (SPM96) (Friston et al., 1995) package.
For the statistical analyses of the neuroimaging data, the general linear model, as implemented by SPM96 (Friston et al., 1995), was used to estimate the blood flow changes between each condition for each voxel. Scan order was covaried using ANCOVA. For activations occurring in areas of the brain previously shown to be involved in performance of the working memory task (ventrolateral and dorsolateral prefrontal, premotor, and posterior parietal cortices), an intensity threshold (uncorrected for multiple comparisons) of $p \leq 0.001$ was applied. For other areas of the brain a corrected intensity threshold of $p \leq 0.05$ was applied. These thresholds, based on 3-D Gaussian random field theory, predict the likelihood of obtaining a false positive in an extended 3-D field.

Behavioral data for the cognitive tasks, cardiovascular measures, and subjective ratings on the visual analog scales were analyzed (using SPSS for the Apple Macintosh) using repeated-measures ANOVAs with session order as a between-subjects factor and drug and, where appropriate, time as within-subjects factors.

\section{RESULTS}

\section{Effects of Ritalin of self-ordered spatial working memory performance}

Analysis of the behavioral performance of subjects confirmed previous findings (Elliott et al., 1997) with subjects on methylphenidate making fewer between-search errors on the SWM task than when on placebo [mean and SEs of search errors per 12 circle problem on drug: 11.5 (3.32) and placebo: 16.1 (4.48); $\left.F_{(1,8)}=5.57 ; p=0.046\right]$. The drug had no effect on within-search errors [drug: 3.24 (1.19) and placebo: $4.95(2.25) ; F_{(1,8)}=1.55$; $p=0.25]$.

\section{Effects of Ritalin on task-dependent and task- independent changes in rCBF}

The effects of methylphenidate on $\mathrm{rCBF}$, independent of task conditions, were analyzed using specific contrasts. Consistent with the effects of methylphenidate on glucose metabolism (Volkow et al., 1997), the drug only produced increases in blood flow in the right cerebellum, but led to decreases in blood flow in regions within the frontal and temporal lobes (Table 1). The loci of task-related effects of methylphenidate on $\mathrm{rCBF}$, as revealed by the significant drug $\times$ task interaction, were clearly defined, with changes in $\mathrm{rCBF}$ in the dorsolateral prefrontal cortex (DLPFC), supplementary motor cortex, and posterior parietal cortex (PPC)all in the left hemisphere (Fig. 1). The adjusted blood flow values for the three regions showing the peak statistical interaction effect between task and drug are displayed in Figure $2 a-c$, exhibited by methylphenidate-induced reductions in $\mathrm{rCBF}$ for the task condi- 
(b)
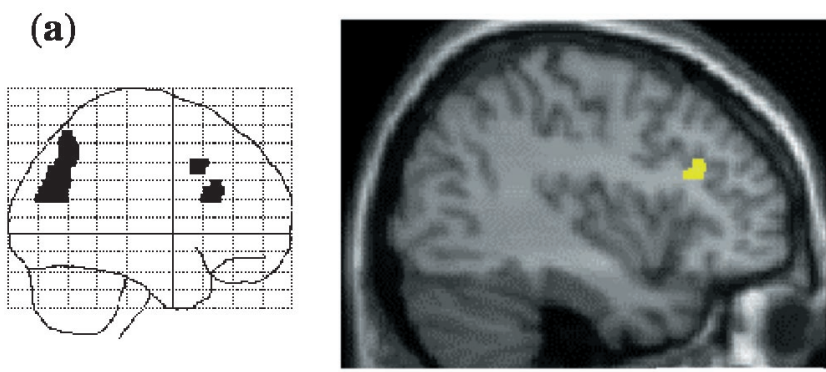

$x=-40$

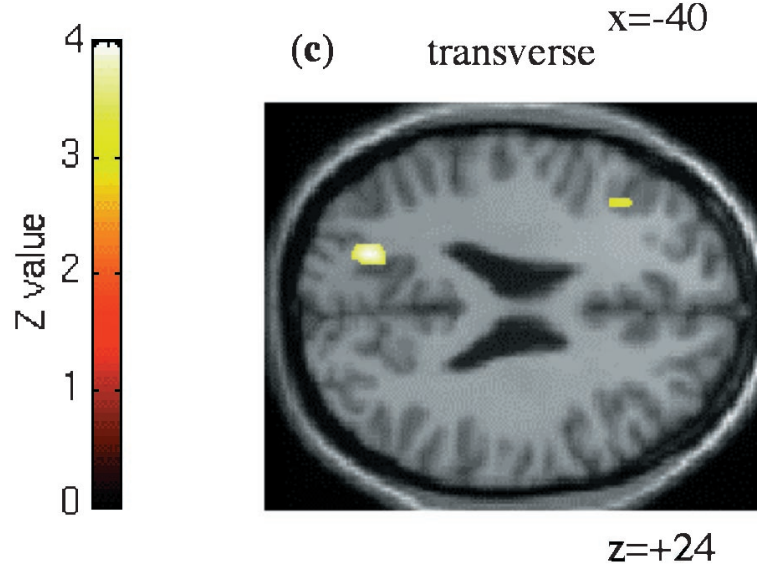

Figure 1. a, Schematic image showing the regions of significant $\mathrm{rCBF}$ change for the interaction effect of the SWM task and methylphenidate (task-control $\times$ drug-placebo) superimposed on an average 3-D Montreal Neurological Institute glass brain using a height threshold of $Z=$ $3.09, p=0.001$. Stereotaxic coordinates $[x, y, z]$ (Table 1, legend), cortical region (Brodmann area), and $Z$ scores for the significant activations (see Materials and Methods) are: $[-40,26,24]$, left dorsolateral prefrontal cortex (9), $Z=3.40 ;[-18,16,40]$, left supplementary motor area (6), $Z=$ 3.69 , and $[-12,-64,48]$, left PPC (7), $Z=3.91 . b$ and $c$ are merged PET-MRI sections illustrating the significant rCBF interaction between drug and task shown in $a$. The interaction effect in the left DLPFC is illustrated in $b$ and $c$; in addition, the interaction in the inferior left PPC is clearly visible in $c$. The front of the brain is to the right in all three images.

tions with no effect of methylphenidate on $\mathrm{rCBF}$ for the control condition. There were no significant interactions of task difficulty with drug (drug-placebo), and so the easy and difficult conditions were combined for the purpose of illustration (Fig. 2). The attenuation of activity in the supplementary motor cortex (Fig. $2 b$ ) was not predicted on the basis of previous findings (Owen et al., 1996b), and because the peak interaction effect $Z$-value (corrected for multiple comparisons) was not significant, it was not analyzed further.

\section{Hemispheric effects of Ritalin on task-dependent changes in rCBF}

Previous research has defined regions within the DLPFC and PPC in the right hemisphere as involved in performance of the self-ordered SWM task. The significant interaction between drug and task in the left hemisphere in the present study was, therefore, surprising, although it is consistent with other cognitive psychopharmacological studies using catecholamine agents (Daniel et al., 1991; Grasby et al., 1992; Mattay et al., 1996). Consequently, supplementary analyses were performed to directly compare the left and right hemisphere drug-induced, taskrelated changes in $\mathrm{rCBF}$ within the DLFPC and the PPC. (a)

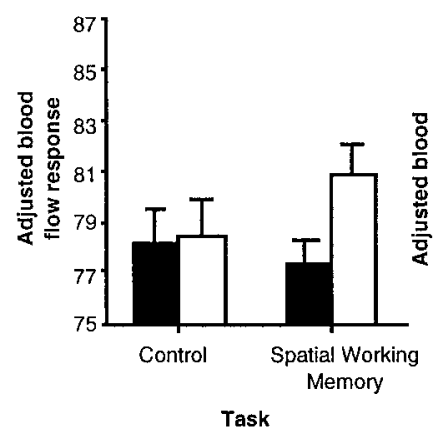

(b)

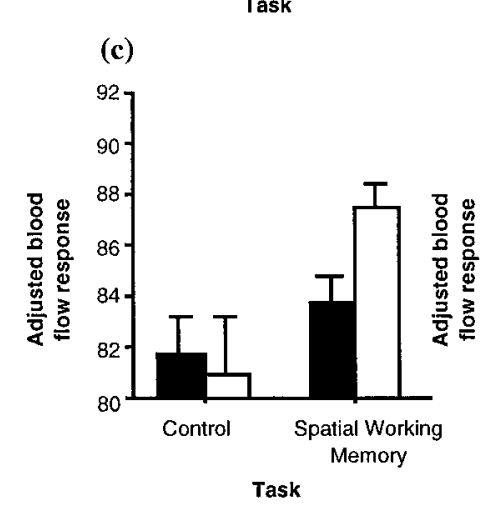

(d)
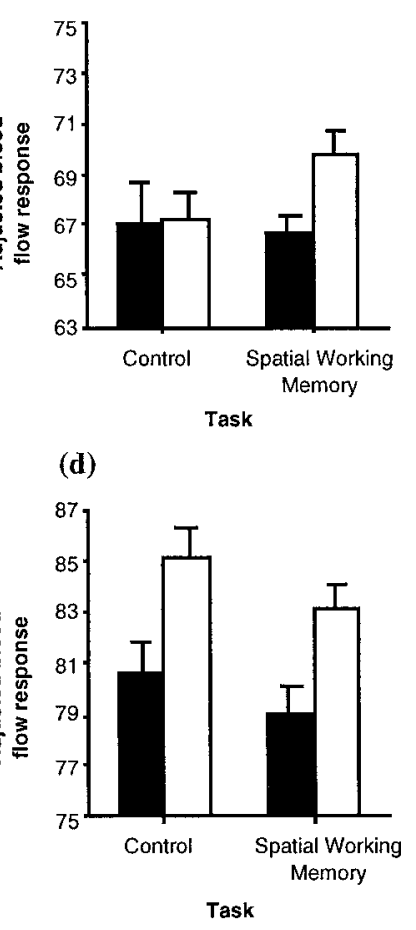

Methylphenidate

Figure 2. Graphical depiction of the task $\times$ drug interaction for mean adjusted rCBF (to the mean global cerebral blood flow) from the peak of statistically significant changes in the left dorsolateral prefrontal cortex $(a)$, left supplementary motor area $(b)$, and left PPC (c). In all three areas there is a methylphenidate-induced reduction in $\mathrm{rCBF}$ for the working memory task condition with no corresponding reduction for the control condition. $d$, Mean adjusted $\mathrm{rCBF}$ for the area of greatest taskindependent, drug-induced reduction in blood flow, the left temporal pole. Values on ordinate are arbitrary numbers of relative adjusted blood flow. Error bars represent SDs.

Repeated-measures ANOVAs showed no interaction of the drug and task with laterality for either the $\operatorname{DLPFC}\left(F_{(1,9)}=2.58 ; p=\right.$ $0.14)$ or the PPC $\left(F_{(1,9)}=2.76 ; p=0.13\right)$. Therefore, it appears that the interaction between methylphenidate and the working memory task, although only reaching significance in the left hemisphere according to our statistical criterion, should not be thought of as being unequivocally unilateral. Indeed, closer inspection of these interactions in the equivalent right hemisphere regions revealed decreases in $\mathrm{rCBF}$ of $Z=1.61$ (DLPFC) and 1.63 (PPC).

\section{Cardiovascular and subjective effects of Ritalin}

Subsequent analyses attempted to specify the underlying cognitive, subjective, or cardiovascular processes that might be associated with the enhancement of SWM performance seen after methylphenidate administration. It was predicted that the cognitive effects of methylphenidate would not be related to druginduced effects on subjective or cardiovascular measures. To assess the influence of methylphenidate on changes in subjective mood ratings, responses on a set of 16 visual analog scales (Bond and Lader, 1974) were analyzed using repeated-measures ANOVAs. Because of the number of comparisons being made, a restricted significance threshold of $p=0.01$ was used. No significant main effects of drug condition or interactions with drug 
Table 2. Mean cardiovascular measures before- and after drug or placebo administration

\begin{tabular}{|c|c|c|c|c|c|c|c|}
\hline & $\begin{array}{l}\text { Pre-drug } \\
\text { pre-scans }\end{array}$ & $\begin{array}{l}\text { Post-drug } \\
\text { mid-scans }\end{array}$ & $\begin{array}{l}\text { Post-drug } \\
\text { post-scans }\end{array}$ & $\begin{array}{l}\text { Pre-placebo } \\
\text { pre-scans }\end{array}$ & $\begin{array}{l}\text { Pre-placebo } \\
\text { mid-scans }\end{array}$ & $\begin{array}{l}\text { Pre-placebo } \\
\text { post-scans }\end{array}$ & SED \\
\hline Heart rate (beats/min) & 64.4 & 62.0 & 65.0 & 66.6 & 64.4 & 69.0 & 0.72 \\
\hline Systolic blood pressure (mmHg) & 121.8 & 145.3 & 142.5 & 125.4 & 133.0 & 131.5 & $1.04 *$ \\
\hline Diastolic blood pressure $(\mathrm{mmHg})$ & 74.0 & 84.0 & 82.2 & 81.0 & 82.0 & 84.1 & 0.91 \\
\hline
\end{tabular}

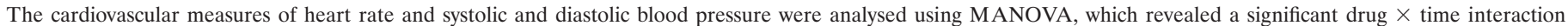

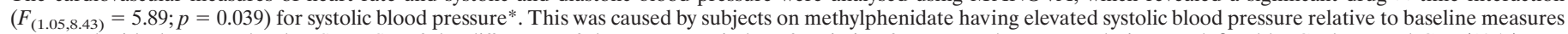

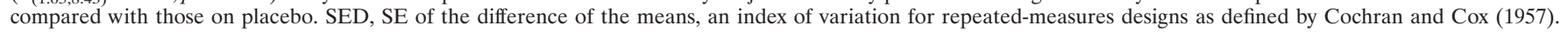

condition were found. Repeated-measures ANOVA was also used to assess the influence of methylphenidate on the cardiovascular measures of heart rate and blood pressure. A significant effect of drug administration was found for the systolic blood pressure measurements (Table 2), but this was not related to the improved performance on the working memory task (Pearson's product moment correlation coefficient, $r=-0.15 ; p=0.68$ ).

\section{Relationship of drug-induced enhanced performance to baseline memory span}

Prompted by the results of a previous study using a dopamine D2 receptor agonist (bromocriptine) (Kimberg et al., 1997), the possible relationship between the baseline memory span scores and the drug-induced improvement in SWM performance was investigated. The drug-induced reduction in SWM errors correlated negatively with verbal $(r=-0.78 ; p=0.007)$, as well as spatial $(r=-0.67 ; p=0.033)$ baseline memory span. Thus, subjects with lower baseline memory capacity demonstrated greater improvement in SWM in terms of error reduction after treatment with methylphenidate. In addition, both the digit and spatial memory span scores were highly, positively, correlated $(r=0.78 ; p=0.008)$, suggesting that the drug-induced improvement may be related to general baseline working memory capacity rather than specifically spatial or verbal components.

\section{The drug $\times$ task interaction effect on rCBF was not attributable to generalized changes in blood flow}

The interaction effects evidenced in this study between methylphenidate administration and SWM task performance were not attributable to generalized effects of methylphenidate on attenuating blood flow (Krimer et al., 1998), because they occurred in different loci than the drug-induced changes in $\mathrm{rCBF}$ (Table 1). It is also unlikely that the significant interactions of methylphenidate and the cognitive task on rCBF were artifacts of the overall drug-induced attenuation of rCBF. This is illustrated by the comparison of Figure $2 d$ to Figure $2 a-c$. Figure $2 d$ shows the adjusted blood flow values for the region of the brain showing the most significant generalized reduction of $\mathrm{rCBF}$ in response to methylphenidate administration - the left temporal pole. In Figure $2 d$ methylphenidate administration led to reduced blood flow in both the working memory and the control tasks. This contrasts with the effects for the left DLPFC, left supplementary motor area, and left PPC (Fig. 2a-c), where reductions in rCBF after methylphenidate were specific to the working memory task conditions. Nor was it the case that all areas of the brain activated by the task were attenuated by methylphenidate. For example, the right ventrolateral prefrontal cortex, which has been shown to be activated by the task (Owen et al., 1996b), shows no significant drug-induced attenuation in $\mathrm{rCBF}$ after drug [mean adjusted blood flow values (with SDs in parentheses) for the stereotaxic coordinates 36,50 , and -20 for control condition and task conditions: on drug, 56.0 (1.47), 56.5 (0.90); on placebo, 56.5 (1.35), 57.7 (1.36)].

\section{DISCUSSION}

The results of this study have demonstrated, we think for the first time, that methylphenidate-induced enhancement of self-ordered SWM performance is accompanied by discrete changes in $\mathrm{rCBF}$ within the DLPFC and PPC. These changes were not caused by generalized effects of methylphenidate on rCBF or artifacts of rCBF changes restricted to cortical regions activated by the task.

The locus of the interaction effect in the left prefrontal cortex is entirely consistent with other neuroimaging studies investigating the neural correlates of monoaminergic modulation of cognitive functions (Daniel et al., 1991; Grasby et al., 1992; Mattay et al., 1996). For example, Grasby et al. (1992) showed that the dopamine agonist apomorphine attenuated increased rCBF during a verbal memory task in the left DLPFC which, unlike our study, was accompanied by impaired performance in those on drug. In the present study, the significant methylphenidateinduced reduction of blood flow in the left DLFPC (and the trend in the right DLPFC) therefore appears important for an understanding of the clear enhancement of performance accuracy on the SWM task. Furey et al. (1997) were able to demonstrate a drug-induced speeding of reaction time (but with no effect on performance accuracy) on a facial recognition task after the anticholinesterase physostigmine. This shortening of response latencies was associated with decreased $\mathrm{rCBF}$ in the right DLPFC (Furey et al., 1997). In contrast, studies with catecholaminergic agents, including the present one, have observed task-related modulation of rCBF in the left DLPFC. Moreover, the present enhancement in performance, induced by methylphenidate, was on choice accuracy, whereas physostigmine shortened response latencies (performance accuracy was at ceiling) in a rather different facial recognition paradigm. There are theoretical grounds for concluding that measures of accuracy and latency reflect different aspects of memory retrieval processes (Baddeley, 1986). Consequently, it would appear that cholinergic and catecholaminergic systems may modulate different aspects of retrieval in the frontal lobes, although further research using a common test procedure would be required to empirically demonstrate such a dissociation.

Because the present task has been shown to preferentially activate the right DLPFC (Owen et al., 1996b), it is possible that the drug-induced improvement in SWM is mediated by effects on other cognitive processes that modulate performance on this task. One possible mechanism may involve a change in task-related rCBF in the left DLPFC associated with a facilitation of verbal rehearsal via the articulatory loop (Baddeley, 1986), given that activation in this area has been observed during some verbal 
working memory tasks (Smith and Jonides, 1998). Indeed, articulatory suppression is known to impair performance in a spatial reasoning task requiring working memory (Vandierdonck and De Vooght, 1997) and in the task used here (T. W. Robbins, A. M. Owen, V. Foot, and J. Newton, unpublished observations), suggesting some verbal mediation of accurate performance.

Alternatively, the drug could be acting to suppress distracting verbal mediation. The lack of evidence for a lateralized effect on $\mathrm{rCBF}$ when the two sides are directly compared, together with the finding of a specific relationship of the drug effect to verbal and visual working memory measures, suggest that the drug may be acting on more general working memory processes. The reduction in activation in the DLPFC on drug during the task performance may be related to methylphenidate treatment resulting in an increased efficiency with which the working memory task is completed by a neural network including the DLPFC. The neuronal nature of this increased efficiency may be manifested as an increased response to signals with concomitant suppression of background noise, a well-documented effect of catecholamines (Foote and Morrison, 1975). Because there were up to 11 distractors present at any stage during the task when the subject was searching for one token, the overall detectable effect of this increase in signal-to-noise ratio may be a reduction in neuronal activity, and hence, a reduction in blood flow in certain regions within the task-specific neural network (Raichle, 1987). The fact that the subjects with the least effective baseline working memory capacity (tested outside the scanner) exhibited the greatest degree of improvement after methylphenidate is consistent with this account (as can be described by sigmoidal functions of signal discriminability) (Kimberg et al., 1997).

A number of psychological processes are thought to be involved in the SWM task (Petrides et al., 1993; see also Goldman-Rakic, 1995) and include (1) online storage and active response organization based on the retrieval of information from posterior cortical association systems (involving the mid-ventrolateral prefrontal cortex), and (2) the active manipulation and monitoring of information within working memory (involving the mid-DLPFC). By analogy, the results of the present study can be taken as reflecting selective modulation of (1) the former processes, mediated by drug-induced changes in $\mathrm{rCBF}$ in the PPC, and (2) the latter processes mediated by drug-induced changes in $\mathrm{rCBF}$ in the left DLPFC. Thus, the findings provide valuable insights into the mechanism of enhancement of SWM performance by the catecholaminergic stimulant drug methylphenidate. These results have important consequences for understanding the methylphenidate-induced improvements seen in children with AD/HD on working memory tasks (Tannock et al., 1995; Kempton et al., 1999) and in comparison with other paradigms (e.g., response inhibition) (Vaidya et al., 1998). More generally, the results are important for understanding the possible beneficial effects of catecholaminergic drugs in a range of cognitive disorders, including acute brain injury (McDowell et al., 1998) and schizophrenia (Mortimer, 1997).

\section{REFERENCES}

Arnsten AFT (1997) Catecholamine regulation of the prefrontal cortex. J Psychopharmacol 11:151-162.

Arnsten AF, Goldman-Rakic PS (1985) Alpha 2-adrenergic mechanisms in prefrontal cortex associated with cognitive decline in aged nonhuman primates. Science 230:1273-1276.

Baddeley AD (1986) Working memory. Oxford: Clarendon.

Bernardi G, Cherubini E, Marciani MG, Mercuri N, Stanzione P (1982) Responses of intracellularly recorded cortical neurons to the iontophoretic application of dopamine. Brain Res 245:267-274.

Bond A, Lader M (1974) The use of analogue scales in rating subjective feelings. Br J Med Psychol 47:211-218.

Cochran WG, Cox GM (1957) Experimental designs. New York: Wiley.

Coull J, Middleton HC, Robbins TW, Sahakian BJ (1995) Contrasting effects of clonidine and diazepam on tests of working memory and planning. Psychopharmacology (Berl) 120:311-321.

Daniel DG, Weinberger DR, Jones DW, Zigun JR, Coppola R, Handel S, Bigelow LB, Goldberg TE, Berman KF, Kleinman JE (1991) The effect of amphetamine on regional cerebral blood flow during cognitive activation in schizophrenia. J Neurosci 11:1907-1917.

Diagnostic and statistical manual of mental disorders, Ed 4 (1994) Washington, DC: American Psychiatric Association.

Elliott R, Sahakian BJ, Matthews K, Bannerjea A, Rimmer J, Robbins TW (1997) Effects of methylphenidate on spatial working memory and planning in healthy young adults. Psychopharmacology (Berl) 131:196-206.

Foote SL, Morrison JH (1975) Effects of putative neurotransmitters on neuronal activity in monkey auditory cortex. Brain Res 86:229-242.

Friston KJ, Holmes AP, Worsley KJ, Poline J-B, Frith CD, Frackowiak RSJ (1995) Statistical parametric maps in functional imaging: a general linear approach. Hum Brain Mapp 2:189-210.

Furey ML, Pietrini P, Haxby JV, Alexander GE, Lee HC, VanMeter J, Grady CL, Shetty U, Rapoport SI, Schapiro MB, Freo U (1997) Cholinergic stimulation alters performance and task-specific regional cerebral blood flow during working memory. Proc Natl Acad Sci USA 94:6512-6516.

Goldman-Rakic PM (1995) Architecture of the prefrontal cortex and the central executive. Ann NY Acad Sci 769:71-83.

Grasby PM, Friston KJ, Bench CJ, Frith CD, Paulesu E, Cowen PJ, Liddle PJ, Frakowiak RSJ, Dolan R (1992) The effect of apomorphine and buspirone on regional cerebral blood flow during the performance of a cognitive task-measuring neuromodulatory effects of psychotropic drugs in man. Eur J Neurosci 4:1203-1212.

Gualtieri C, Wargin W, Kanoy R, Patrick K, Shen CD, Youngblood W, Mueller RA, Breese GR (1982) Clinical Studies of methylphenidate serum levels in children and adults. J Am Acad Child Psychiatry 2:19-26.

Kempton S, Vance A, Maruff P, Luk E, Costin J, Pantelis C (1999) Executive function and attention deficit hyperactivity disorder: stimulant medication and better executive function performance in children. Psychol Med 29:527-538.

Kimberg DY, D'Esposito M, Farah MJ (1997) Effects of bromocriptine on human subjects depends on working memory capacity. NeuroReport 8:3581-3585.

Krimer LS, Muly EC, Williams GV, Goldman-Rakic PS (1998) Dopaminergic regulation of cerebral cortical microcirculation. Nat Neurosci $1: 286-289$.

Mattay VS, Berman KF, Ostrem JL, Esposito G, Van Horn JD, Bigelow LB, Weinberger DR (1996) Dextroamphetamine enhances "neural network-specific" physiological signals: a positron-emission tomography rCBF study. J Neurosci 16:4816-4822.

McDowell S, Whyte J, D'Esposito M (1998) Differential effect of a dopaminergic agonist on prefrontal function in traumatic brain injury patients. Brain 121:1155-1164.

Mortimer AM (1997) Cognitive function in schizophrenia- do neuroleptics make a difference? Pharmacol Biochem Behav 56:789-795.

Owen AM, Morris RG, Sahakian BJ, Polkey CE, Robbins TW (1996a) Double dissociations of memory and executive functions in working memory tasks following frontal lobe excisions, temporal lobe excisions or amygdalo-hippocampectomy in man. Brain 119:1597-1615.

Owen AM, Evans AC, Petrides M (1996b) Evidence for a two-stage model of spatial working memory processing within lateral frontal cortex: a positron emission tomography Study. Cereb Cortex 6:31-38.

Petrides M, Alivisatos B, Evans A, Meyer E (1993) Dissociation of human mid-dorsolateral from posterior dorsolateral frontal cortex in memory processing. Proc Natl Acad Sci USA 90:873-877.

Raichle ME (1987) Circulatory and metabolic correlates of brain func- 
tion in normal humans. In: Handbook of physiology: the nervous system $\mathrm{V}$ : higher functions of the brain (Plum F, ed), pp 643-674. Bethesda, MD: American Physiological. Society.

Riordan HJ, Flashman LA, Saykin AJ, Frutiger SA, Carroll KE, Huey L (1999) Neuropsychological correlates of methylphenidate treatment in adult ADHD with and without depression. Arch Clin Neuropsychol 14:217-233.

Sawaguchi T, Matsumura M, Kubota K (1988) Dopamine enhances neuronal activity of a spatial short-term memory task in the primate prefrontal cortex. Neurosci Res 5:465-473.

Seeman P, Madras BK (1998) Anti-hyperactivity medication: methylphenidate and amphetamine. Mol Psychiatry 3:386-396.

Scheel-Krüger J, Braestrup C, Nielson M, Golembiowska K, Mogilnika E (1977) Cocaine: discussion on the role of dopamine in the biochemical mechanism of action In: Cocaine and other stimulants (Ellinwood $\mathrm{Jr} \mathrm{H}$, Kilbey MM, eds). New York: Plenum.
Smith EE, Jonides J (1998) Neuroimaging analyses of human working memory. Proc Natl Acad Sci USA 95:12061-12068.

Tannock R, Ickowicz A, Schachar R (1995) Differential effects of methylphenidate on working memory in ADHD children with and without comorbid anxiety. J Am Acad Child Adolesc Psychiatry 34:886-896.

Vaidya CJ, Austin G, Kirkorian G, Ridlehuber HW, Desmond JE, Glover GH, Gabrieli JDE (1998) Selective effects of methylphenidate in attention deficit hyperactivity disorder: a functional magnetic resonance study. Proc Natl Acad Sci USA 95:1494-14499.

Vandierdonck A, De Vooght G (1997) Working memory constraints on linear reasoning with spatial and temporal contents. Q J Exp Psychol A 50:803-820.

Volkow ND, Wang GJ, Fowler JS, Logan J, Angrist B, Hitzemann R, Lieberman J, Pappas N (1997) Effects of methylphenidate on regional brain glucose metabolism in humans: relationship to dopamine D2 receptors. Am J Psychiatry 15:50-55. 